\title{
Catalogue of Life Plus: innovating the CoL systems as a foundation for a clearinghouse for names and taxonomy
}

\author{
Olaf Bánki ${ }^{\ddagger}$, Markus Döring ${ }^{\S}$, Ayco Holleman ${ }^{\ddagger}$, Wouter Addink ${ }^{\ddagger}$ \\ ‡ Naturalis Biodiversity Center, Leiden, Netherlands \\ $\S$ GBIF, Copenhagen, Denmark
}

Corresponding author: Olaf Bánki (olaf.banki@naturalis.nl)

Received: 24 May 2018| Published: 28 May 2018

Citation: Bánki O, Döring M, Holleman A, Addink W (2018) Catalogue of Life Plus: innovating the CoL systems as a foundation for a clearinghouse for names and taxonomy. Biodiversity Information Science and Standards 2 : e26922. https://doi.org/10.3897/biss.2.26922

\section{Abstract}

In 2015, the global biodiversity information initiatives Biodiversity Heritage Library (BHL), B arcode of Life Data systems (BoLD), Catalogue of Life (CoL), Encyclopedia of Life (EOL), and the Global Biodiversity Information Facility (GBIF) took the first step to work on the idea for building a single shared authoritative nomenclature and taxonomic foundation that could be used as a backbone to order and connect biodiversity data across various domains. At present, the Catalogue of Life is being used by BHL, BoLD, EOL, and GBIF, but each extend the CoL with additional data to meet the specific backbone services required.

The goal of the CoL+ project is to innovate the CoL systems by developing a new information technology infrastructure that includes both the current Catalogue of Life and a provisional Catalogue of Life (replacing the current GBIF backbone taxonomy), separates scientific names and taxonomic concepts with associated unique identifiers, and provides 
some (infrastructural) support for taxonomic and nomenclatural content authorities to finish their work. The project's specific objectives are to

1. establish a clearinghouse covering scientific names across all life; provide a single taxonomic view grounded in the consensus classification of the Catalogue of Life along with candidate taxonomic sources, show differences between sources, and provide an avenue for feedback to content authorities while allowing the broader community to contribute, and

2. establish a partnership and governance, allowing a continuing commitment after the project's end for a clearinghouse infrastructure and its associated components, including a roadmap for future developments of the infrastructure.

As result of the project we expect to have a shared information space for names and taxonomy between the Catalogue of Life, nomenclator content authorities (e.g. IPNI, ZooBa nk) and several global biodiversity information initiatives.

\section{Keywords}

Catalogue of Life, nomenclature, taxonomy, species checklist

\section{Presenting author}

Wouter Addink

\section{Presented at}

SPNHC+TDWG 2018

\section{Acknowledgements}

As part of the GBIF global work programme, the Netherlands government, through the Net herlands Biodiversity Information Facility, provided a grant of $€ 360 \mathrm{~K}$ for a two year CoL+ project (2017-2019). The Catalogue of Life, Naturalis Biodiversity Center, and the Global Biodiversity Information Facility complemented this with in-kind contributions for a total amount of $€ 728 \mathrm{~K}$. 